\title{
Assessment of Cost Monitoring System (Pune Real Estate Sector)
}

\author{
Shreyash Raut ${ }^{1}$, Dr S.S. Pimplikar ${ }^{2}$ \\ ${ }^{I}$ Student, M.E. (Construction \& Management), Maharashtra Institute of Technology, Pune, India. \\ ${ }^{2}$ Professor \& Head, Civil Engg. Dept, Maharashtra Institute of Technology, Pune, India
}

\begin{abstract}
It is very common to see project failing to achieve its mission within specified time and cost. The factors contributing to overrun are inadequate project formation, poor planning for implementation and lack project management during project execution but the main cause of failure can be attributed to cost estimation failure and management failure. As project become larger and more complex, the ability to exchange information on a timely basis is shrinking. Looking to current scenario in Pune construction industry problem faced is of cost \& time overruns. This paper is intended at exploring the cost monitoring technique used to manage the budget of the real estate construction projects in Pune. Suggesting feasible monitoring methods to the suitable for pune's real estate sector working environment \& approach of the industry towards construction projects is also one of the intentions of these papers.
\end{abstract}

Keywords: CAD drawings, Cost Management, Digital techniques, ERP-SAP, EVA

\section{Introduction}

Pune has witnessed tremendous growth and development in real estate in the recent years. The city continues to witness good traction this year as well as this market continues to be relatively more affordable and new launches are helping to keep the momentum. Real estate in Pune has grown more rapidly and to higher levels than in most other Indian cities. Property prices have multiplied in almost all parts of the city and the suburbs and neighbouring areas have been getting absorbed at a considerable pace - although the market is not fully immune to a slowdown in the global and national economic environment.

The Pune real estate market has been driven by genuine demand resulting in increased development of both commercial as well as residential space. The real estate sector in Pune has seen a lot of deviations, initially dominated by commercial players to residential groups now. The market was earlier dominated by commercial real estate projects but with the increasing number of immigrants and working population the demand for residential housing projects has increased tremendously.

There are various reasons for Pune's sudden rise in real estate fortunes. Pune is home to a large number of educational and training institutions that cover every aspect of academics, finding such a diverse treasure of knowledge and learning in one city is quite unusual. Pune also boasts of other attractions to tourists. For instance, it offers a full-fledged horse racing infrastructure which is a major attraction among people from other cities. However, the major driving force behind Pune's success in real estate market is the Information Technology edge. Pune offered big IT companies the required space and infrastructure they needed. The big IT giants were looking for huge space for building their offices as well as residential complexes for their employees; Pune sufficed their requirements and offered them a high level of comfort, security and civic amenities. At present, the real estate and construction sectors are playing a crucial role in the overall development of Pune's core infrastructure. Pune real estate industry's growth is primarily linked to developments in the retail, hospitality, Special Economic Zones (SEZs) and IT Industry.

\section{Aims \& Objectives}

This paper is intended at exploring the cost monitoring technique used to manage the budget of the real estate construction projects in Pune. Suggesting feasible monitoring methods to the suitable for pune's real estate sector working environment $\&$ approach of the industry towards construction projects is also one of the intentions of this paper.

\section{Research Methodology}

A questionnaire survey form was designed to collect data. It consists of respondent from several real estate companies in Pune. Data had been collected by face to face interview \& by getting draft of questionnaire filled through e-mails. The total number of responses received back were 29 out of 35 .The purpose of this quantitative data is to get general Project Information and Information Regarding cost Monitoring awareness \& technique used by Pune real estate sector companies and suggesting the new approach. The sample questionnaires for quantitative data of General Project Information and Information Regarding cost Monitoring is given as following: 
1. What cost monitoring system is used in your organisation?

2. Reason to use stated cost monitoring system?

3. Which of the following cost monitoring system you are aware of the following:

- EVA by MSP/Primavera

- Conventional Methods(inflow/outflow)

- Digital Project /cost monitoring tool

i.3D sensing

ii.Digital Hardhat system

iii.Photonet II

iv.SKALA

v.Simulation Model

- $\quad$ Project Monitoring Tool-SAP

4. Which Financial planning \& controlling systems are being used? How they are linked to your work plan?(

Excel, Tally, other ERP system-names)

5. What is the importance given to have a specific monitoring system in your organisation:

- Very less

- Less

- Average

- High

- Very High

6. What is the extend of budget your organisation is ready to spend to install or implement a good monitoring system

- Very less ( upto 5 Lacs)

- Less ( Upto 10 Lacs)

- Average ( upto 25 Lacs)

- $\quad$ High (Upto 50 Lacs)

- Very High ( Above 50 Lacs)

7. What are the Objectives to use stated cost monitoring system? How does it functions ( flow chart)? What are inputs required?

8. What is the Fruitfulness of system? How does it useful in cost monitoring, Anticipation of financial risks \& control over such risks?

9. Does it help to complete the project in time \& within the budget?

10. The importance given to have a specific monitoring system in your organisation:

- Very less

- Less

- Average

- High

- Very High

\section{Findings}

According to questioner survey following were the cost monitoring systems used by several real estate organisations in Pune.

TABLE 1: Response Of Respondent

\begin{tabular}{|c|l|c|}
\hline Sr.No. & \multicolumn{1}{|c|}{ Cost monitoring System } & $\begin{array}{c}\text { No of } \\
\text { respondent }\end{array}$ \\
\hline 1 & EVA By MSP/Primavera & 18 \\
\hline 2 & Conventional Methods & 4 \\
\hline 3 & Digital Project/Cost monitoring tool & 0 \\
\hline 4 & Project monitoring tool-SAP & 7 \\
\hline
\end{tabular}

- It is seen that Earned value analysis is the basic tool used for cost monitoring by real estate sector in Pune.

- EVA is suitable for the organisation as planning schedule work is done on MS project so as results of EVA is easily generated.

- In conventional methods inflow and outflow records are kept for cost monitoring only in Small medium enterprises. 
- Digital project cost monitoring system does not seem feasible for majority of the construction organizations in Pune. In fact it was observed that most of the construction professionals are not even aware about the existence of any such project monitoring system. Further the installation process and operating process of this system will need highly trained and skilled personnel. This cost of installation and operating will also be out of the capacity of the firms.

- ERP-SAP is recommended to use for financially strong organisations. Since there is no need to separately manage resources \& create its own database so as beneficial to use for new projects.

\subsection{EARNED VALUE ANALYSIS}

\section{Cost Monitoring Techniques}

EVA defines and monitors three key dimensions, which are defined as follows:

- Planned value (PV): PV is the authorized budget assigned to the work to be accomplished for an activity or work breakdown structure.

- Earned value (EV): EV is the value of work performed expressed in terms of the approved budget assigned to that work for an activity or work breakdown structure component.

- Actual cost (AC): AC is the total cost actually incurred and recorded in accomplishing work performed for an activity or work breakdown structure component. It is the total cost incurred in accomplishing the work that the EV measured.

Cost Variance (CV) helps to measure project performance. Cost variance $(\mathrm{CV})$ indicates how much over or under budget the project is. A positive value indicates a favourable condition and a negative value indicates an unfavourable condition.

$$
C V=E V-A C
$$

Schedule Variance (SV) indicates how much ahead or behind schedule the project is. A positive value indicates a favourable condition and a negative value indicates an unfavourable condition.

$$
S V(\text { Schedule Variance })=E V-P V \text {. }
$$

CPI (Cost Performance Index) is a measure of cost efficiency on a project. A value equal to or greater than one indicates a favourable condition and a value less than one indicate an unfavourable condition.

$$
\mathrm{CPI}=\mathrm{EV} / \mathrm{AC}
$$

If $\mathrm{CPI}<1$ : The project is running over budget,

If $\mathrm{CPI}>1$ : The project is running under budget,

If $\mathrm{CPI}=1$ : the project is running on budget.

SPI (Schedule Performance Index) is a measure of schedule efficiency on a project. An SPI equal to or greater than one indicates a favourable condition and a value of less than one indicate an unfavourable condition.

$$
\mathrm{SPI}=\mathrm{EV} / \mathrm{PV}
$$

If SPI < 1: The project is running behind planned schedule,

If SPI $>1$ : The project is running ahead of planned schedule,

If $\mathrm{SPI}=1$ : the project is running on planned schedule[3].

EVA helps to know the value of work completed at any given point .This helps to identify deviation of the actual cost from the planned value. Based on this, EVA helps to predicts the possible budget required at the end of the project.EVA doesn't need any special software \& skills to operate .Basic trainings of earned value \& variance calculations can help to monitor the cost of project. It can thus be effectively implemented in any organisations, ranges to local SMES to MNC.

\subsection{D-4D PROGRESS TRACKING TECHNIQUES}

Using new technologies in construction has been shown in several research efforts to improve productivity in construction projects and as a result, save time and cost. This technique combines 3D imaging, object recognition, and 4D modelling technologies to track construction project progress. The automated progress tracking system developed here was built upon the automated object recognition system by adding construction schedule information as the fourth dimension. Given a laser scan of a construction site and its acquisition date, the system quasi-automatically recognizes the building elements that are expected to be built at that date and visible in the scan. Results from multiple scans obtained on the same date but from different 
locations can be aggregated, and the combined recognition results are used to automatically infer site progress status, and subsequently update the schedule. This system was tested with real life data acquired over the course of construction of the Engineering V Building at the University of Waterloo. Experimental results demonstrate the significant potential of this system for automated 3D progress tracking, which should result in improved construction productivity, as well as improved schedule and cost performance for the Canadian construction industry. This technique consists of following stages:

- Design and Implementation Stage.

$\circ$ Design of the progress tracking and schedule updating algorithms

- Implementation of the algorithms

- Field data acquisition.

- Analysis of field data to validate the tracking approach.

This combines three dimensional (3D) point clouds with project 3D CAD model and schedule information to track construction progress. On one hand, 3D laser scan data provides current site conditions. On the other, the 3D CAD model combined with schedule information (the project 4D model), provides designed (as-planned) spatial characteristics of the facility under construction over time. Using such a 4D model, a timestamped 3D CAD model can thus be formed automatically for a given date. The proposed system for automated progress tracking and schedule updating requires the 3D point clouds and the $4 \mathrm{D}$ model to be registered in the same coordinate system to be able to extract useful data for progress tracking. Once registered, as-built objects can be recognized, progress estimated, and the schedule updated all automatically.

For this techniques high precision is to be maintained by using Three dimensional (3D) sensing technologies, such as 3D laser scanners (LADARs) and photogrammetric [5]. This technique is not been feasible for real estate sector in pune as there no such favourable conditions to use them.

\subsection{DIGITALIZED CONSTRUCTION MONITORING (DCM) MODEL}

Digitalized Construction Monitoring (DCM) Model issued for monitoring and evaluating the physical progress of the project. This system is digitalizing the project progress monitoring and proposing prototype software, which integrate the AutoCAD drawings and digital images.

DCM prototype system integrates the information from construction drawings, digital images of construction site progress and planned schedule of work. Using emerging technologies and information system the DCM re-engineer the traditional practice for monitoring the project progress. This system can automatically interpret $\mathrm{CAD}$ drawings of buildings and extract data on its structural components and store in database. It can also extract the engineering information from digital images and when these two databases are simulated the percentage of progress can be calculated and viewed in Microsoft Project automatically. The application of DCM system for monitoring the project progress enables project management teams to better track and controls the productivity and quality of construction projects. The use of the DCM can help resident engineer, construction manager and site engineer in monitoring and evaluating project performance. The simulation concept of DCM model is currently being used to test and check the validity. The main goal of DCM model is to propose an interface process model between the $2 \mathrm{D}$ digital photo and detail design drawings and update the physical progress chart by integrating the information.

This model will improve decision-making process and provides better mechanism for advanced project management [6].

\subsection{PHOTO NET II}

Abeid et al. (2003) developed an automated real-time monitoring system PHOTONET II (so-called web-based system) for construction projects programmed in a Delphi Environment (object-oriented Pascalbased visual programming language). This system links time-lapse digital movies of construction activities, critical path method (CPM) and progress control techniques to show the planned versus actual schedules. PHOTO-NET II connects archived film clips to schedule and progress information to minimize the occurrence of construction disputes. This system uses the Internet as a communication medium between the cameras and the remote computer(s) and the ability to control the playback frame rate when playing back the time-lapse movies. In the end product this system displayed two dynamic diagrams:

- The planned versus actual schedules in a bar chart format;

- The planned versus actual percentage of completion in a histogram format [1].

Cheung et al. (2004) described a Web-based construction project performance monitoring system (PPMS) to exercise the construction project control and as a project monitoring tool. The smooth functioning of the PPMS relies heavily on the internet and the databases system [2]. 


\subsection{SKALA}

SKALA is a web based project monitoring for PWD Malaysia and was first developed in-house by PWD Information and Communication Technology (ICT) Branch. Although there are other software's applications for project management system, the uniqueness of SKALA is that it is tailored to PWD's certified ISO 9001 Quality Management System processes. It was developed to customise the needs of PWD's main core business which is project Management. The main function of SKALA system to generate real time project status for all PWD personnel as well as its client. The major problem on system integration \& collaboration in the construction industry is difficult to access accurate data, information and knowledge in timely manner in every phase of construction project lifecycle. SKALA is an official online project monitoring web page for PWD Malaysia and was first developed in-house by PWD Information and Communication Technology (ICT) Branch in 1985. It was developed and customized to cater the needs of PWD's main core business which is project management. SKALA data input is based on the workflow of PWD business processes which is governed by PWD's certified ISO 9001 Quality Management System. In other words, the users' understanding of PWD's business processes are handled by its Standard Quality Management System while SKALA only automates all critical processes such as project planning documentation, design quality plan, construction quality plan and standard PWD's contract administration forms[4].

\subsection{ERP-SAP}

A The System Application Product (SAP) is integrated Enterprise Resource Planning (ERP) software used for how to plan business resources (materials, employees, customer etc) are acquired \& processed. There are various modules used such as, Project system (PS), Material Management (MM), Sales \& distribution (SD), Finance \& Control (FICO), Plant \& Maintenance (PM \& QM) \& Document Management System (DMS).SAP creates its own database having all ongoing and completed projects interlink with a server so as one can get a reference of completed projects so as to estimate or budget the new project.

SAP has unique code for material in is same for all his project so it become easy to analysis and generate report or track down time material required, to be purchased and also reconciliation for the same becomes easy. Day to day updates from site is done of total work complete and material issue for the same. So the actual track of project completed is known also total inventory used under assigned is been noted. SAP helps to know the total inventory required for the next rolling period. So required funds are as per arrange for the project. The process of SAP consist of the following steps-

- Project Creation.

- Project Date Planning

- Preliminary Costing

- Budgeting \& Release

- Actual Costing.

- Release of Project.

- Project Monitoring-Time, Material variance, Cost Variance \& Reports.

5.6.1 Project Creation:-It is initial step of creating a project in a SAP database. The project has an unique ID number. If project consist of a cluster of building each every building is created as separate projects. Project creation consist of following steps-

- Creation of WBS

- Creation of network

- Creation of activity

- Creation of milestones

5.6.1.1 Creation of WBS:-Creation of work breakdown structure shows collective cost of activities. Operative indicator in WBS consist elements of planning, accounts \& billing. The purpose of element is such that when you tick on planning element you can plan a cost. If you tick on account you can post the cost. Whereas when billing is activated you can plan as well as post the cost.

5.6.1.2 Creation of Network:-In network activities are attached as per WBS. Network helps us in such a way that you can work on same project at same time in different network. Creation of network includes Network description, MRP controller, Type of scheduling.

5.6.1.3 Creation of activity:-In this activities to be carried out is been attached with their normal durations \& material required .Here internal activity means activities which are done internally for example company excavator will do excavation in these case you can attached material but not any labor. Internal activity is used for asset planning. In internal activity inputs are required such as, work center, work etc. Work center field is used to join type of equipment, which is maintained by company. Work is used to join the total working time of equipment which it will spend on project. 
5.6.1.4 Creation of Milestones:-Milestones are assigning to activities for client billing purpose. Following are the inputs given such as milestone description, sales document date, invoice percentage etc.

5.6.2 Project Date Planning:-After creation of activities there comes with linking of activities for project scheduling. After date planning we have to make a project version for delay monitoring with our original dates. Project version is nothing but a snapshot of the project at a time of making project version. Upload the project from MS Project to SAP PS module. While uploading should take care that Number (ID) of activity should be unique, there should not be any constraints for activity in MS project $\&$ start date of project is aligned with first activity in bar chart.

5.6.3 Preliminary Costing:-From this step the actual process to study cost management in SAP starts. In SAP preliminary cost planning is done at three different levels. Three different $\mathrm{CO}$ Versions will be created as $0,1, \& 2$ for cost planning. Preliminary cost planning will be in CO version 1.Detailed cost planning will be in $\mathrm{CO}$ version 2 based on $\mathrm{BPO}$, where as network cost planning will be in $\mathrm{CO}$ version 0 i.e. assigning material \& services. The Cost planning of $\mathrm{CO}$ Version 1 is done based on previous experience \& constants. After receiving the drawings cost planning of $\mathrm{CO}$ version 2 is done. Base planning object is prepared for unit quantity of work. We can join material, labor, equipment, variable for unit of work. BPO is only used for preliminary cost planning. It is just like rate analysis for a item. This preliminary costing helps to get the total project expenditure and based on previous experience we get scope for cost optimization.

5.6.4 Project Budgeting \& Release:- Budget is the approved cost framework for the project and indicates the funds available for the project. Cost planning done in $\mathrm{CO}$ version $1,2 \& 0$ will be converted in to budget after approval from management. Project System enables releasing the Budget periodically \& partly. Availability Control prevents the assignment of funds in excess of the released Budget or current Budget. Availability Control will trigger warnings for every transaction if the total assigned value exceeds $100 \%$ of the given Budget for a particular WBS Element and can even stop further transactions once $110 \%$ of the Budget is exceeded. Original budget are made only once. Original budget can be updated in the form of supplement, return or transfer of budget.

5.6.5 Project Actual Costing:-In SAP actual project costing is done at the stage when we get all concern final drawing for particular activities. This is actual costing based on present constant, activity quantity, its present labor rate etc. Actual costing is done in COST VERSION (CO) " 0 ”. Here joining actual material \& services to the activities is done to get actual project cost.

5.6.6 Release of Project: WBS or activity can be independently released. After release of activity it is opened for execution that is for material procurement, service procurement \& confirmation from site. Reservations for stock items, purchase requisitions for non stock items \& services are raised immediately. Major advantage of SAP is for material management. In overall project cost $70 \%$ contributes for material cost. When it able to track down the required material for next rolling period it helps to get material in time on site.

5.6.7 Project Monitoring:-Project monitoring is tracking of activities day to day with help of data updated in system. It is purely execution phase. Project monitoring consist tracking of following:

5.6.7.1 Project Scheduling- After entering actual dates from site for day to day we have to schedule the project to have effect of this actual date. The whole project is rescheduled as per the actual dates. This will effect to your material requirement plan \& cash outflow.

5.6.7.2 Time Monitoring-In order to track down time as per scheduling done after actual dates received from site so as to get effect in project completion. For each individual activity actual finish, early start \& early finish is updated.After rescheduling as per site current data new finish dates of project activity is known. Time monitoring is done with base plan versus current reschedule plan.

5.6.7.3 Material Variance Monitoring- Material variance is track down with the quantity of material required (reservation) among the quantity used. SAP help easily to track by generating report as material available in store and also helps to known the material required for next month rolling plan. It avoids the material to get delay on sites. Material variance with their cost effect on total saleable cost is also be track which helps to an option for cost optimization.SAP has added advantage if any project lack with specific material management can easily track for which site that material is excess which can be transferred and avoid delay in such material activity. Material variance monitoring helps to analysis total inventory cost overflows. 
5.6.7.4 Cost Monitoring-Cost monitoring is done with material and services (labor) cost. Cost monitoring is done with original budget versus current budget versus Estimate to complete.

ERP-SAP reduces the paper work and is easy access such that any personal in organisation can track down work in his scope.SAP major advantage is it mange the inventory in such a manner that minimum possible problems arises for material to reach on site within its lead time. Top management gets the MIS of all sites with a report at his figure tip. The major requirement is training required for staff members so has to be punctual and reliable about the correct day to day activity update. Cost required is more to implement SAP in an organisation but quit beneficial for a financial strong organisation so as to track down multiple site. SAP helps to track down the cash flow \& time effectively with added advantages.

\section{Conclusion}

From this study the following conclusions of monitoring Systems for pune real estate sector can be drawn,

- EVA is the basis for all the software monitoring systems. Most of medium size construction firms in Pune use EVA through project management software's.

- ERP-SAP seems to be an emerging cost \& project monitoring system among financial strong organizations.

- ERP-SAP has been proved to be a good management information system for an organization so as to keep the track on multiple ongoing projects in Pune.

- Digitalized Construction Monitoring system does not seem feasible for Pune real estate owing to its complexity, high installation \& operating cost, requirement of trained and skilled personnel etc.

\section{References}

[1] Abeid, J.N., Allouche, E., Arditi, D. and Hayman, M.,PHOTO-NET II: a computer-based monitoring system applied to project management. Automation in construction, ASCE (2003). 12(5): 603-616.

[2] Cheung, S.O., Suen, H.C.H. and Cheung, K.K.WPPMS: a web based construction Project Performance Monitoring S y s t e m, Automation in construction 13 (2004):361-376

[3] Mohd Faris Khamidi and Waris Ali Khan, "The cost monitoring of construction projects through earned value analysis", International conference on economics and finance research,Singapore,2011.

[4] Redzuan Bin Jaafar,' Collabaration and Communication Of Project Team In PWD Web Based Project Monitoring System 'University teknologi,Malaysia.

[5] Yelda Turkan,"Automated Construction Progress Tracking using 3D Sensing Technologies", A thesis presented to the University of Waterloo,Canada 2012.

[6] Zubair Ahmed Memon, Mudh. Zaimi Abd. Majidand Mushairry Mustaffar, 'A Systematic approach for monitoring and Evaluating the construction project progress' Journal-The Institution of Engineers, Malaysia (vol. 67,No. 3, September 2006). 